\title{
Reverse Bayesianism and Act Independence
}

Surajeet Chakravarty

University of Exeter

David Kelsey

University of Exeter, University of Nottingham

Joshua C. Teitelbaum

Georgetown University Law Center, jct48@law.georgetown.edu

This paper can be downloaded free of charge from:

https://scholarship.law.georgetown.edu/facpub/2263

https://ssrn.com/abstract=3605584

Journal of Economic Theory, forthcoming.

This open-access article is brought to you by the Georgetown Law Library. Posted with permission of the author. Follow this and additional works at: https://scholarship.law.georgetown.edu/facpub

3 Part of the Law and Economics Commons 


\title{
Reverse Bayesianism and Act Independence*
}

\author{
Surajeet Chakravarty \\ University of Exeter
}

\author{
David Kelsey \\ University of Nottingham
}

\author{
Joshua C. Teitelbaum \\ Georgetown University
}

Draft: December 21, 2021

\begin{abstract}
Karni and Vierø (2013) propose a model of belief revision under growing awarenessreverse Bayesianism - which posits that as a person becomes aware of new acts, consequences, or act-consequence links, she revises her beliefs over an expanded state space in a way that preserves the relative likelihoods of events in the original state space. A key feature of the model is that reverse Bayesianism does not fully determine the revised probability distribution. We provide an assumption-act independence - that imposes additional restrictions on reverse Bayesian belief revision. We show that with act independence knowledge of the probabilities of the new act events in the expanded state space is sufficient to fully determine the revised probability distribution in each case of growing awareness. We also explore what additional knowledge is required for reverse Bayesianism to pin down the revised probabilities without act independence.
\end{abstract}

JEL codes: D83, K23.

Keywords: act independence, reverse Bayesianism, unawareness.

\footnotetext{
${ }^{*}$ Corresponding author: Joshua C. Teitelbaum, Georgetown University, 600 New Jersey Avenue NW, Washington, DC 20001 (jct48@georgetown.edu). We thank the editor and the referees for their helpful comments. We also thank Jonah Gelbach, Tim Friehe, Simon Grant, John Mikhail, David Reinstein, Steve Salop, Steve Shavell, Larry Solum, Marie-Louise Vierø, and participants at the following conferences and seminars: the Law and Ambiguity Workshop at the University of Exeter, the Decisions, Markets, and Networks conference at Cornell University, the 34th Annual Conference of the European Association of Law and Economics (EALE), Bielefeld University, Towson University, Université Paris Nanterre, Universität Bonn, the 2018 Annual Meeting of the American Association of Law Schools (AALS) Section on Law and Economics, the 28th Annual Meeting of the American Law and Economics Association (ALEA), the 2018 Foundations of Utility and Risk Conference (FUR), the 8th Annual Law and Economics Theory Conference (LET), Australian National University, University of Auckland, and University of Nottingham.
} 


\section{Introduction}

Overview. Economists traditionally model choice under uncertainty according to Savage's theory of subjective expected utility (Savage, 1954). Savage's theory posits a space of mutually exclusive and collectively exhaustive states of the world, representing all possible resolutions of uncertainty. It assumes that when a person chooses an act, although she is uncertain about the true state of the world and therefore about the consequences of her chosen act, she nevertheless has complete knowledge of the state space she knows all the possible acts and all the possible consequences of each and every act.

In reality, however, a person often does not have complete knowledge of the state space. This is known as unawareness. A person may be unaware of some acts, some consequences, or that a known act can cause a known consequence. Unawareness creates the possibility of growing awareness - the expansion of the state space when a person discovers a new act, consequence, or act-consequence link. Examples of growing awareness include the discovery of a new product or technology (new act), the discovery of a new disease or injury (new consequence), or the discovery of a new link between a known product and a known injury (new act-consequence link). ${ }^{1}$

"Unawareness refers to the lack of conception rather than the lack of information" (Schipper, 2014a,b). There is a fundamental difference between not knowing the state of the world (lack of information) and not knowing that a state of the world is possible (lack of conception). The Savage model allows the state space to contract with the arrival of information and is consistent with Bayesian updating of beliefs. It however does not admit unawareness and cannot accommodate growing awareness (Dekel et al., 1998a,b).

\footnotetext{
${ }^{1}$ For instance, the development of modern day hydraulic fracturing, or "fracking," in the late 1990s (Gold, 2014) (new act); the discovery of HIV/AIDS in the early 1980s (U.S. Centers for Disease Control and Prevention, 2011) and bovine spongiform encephalopathy, or "mad cow disease," in the late 1980s (Collee and Bradley, 1997) (new consequences); and the discovery of links between Agent Orange and cancer after the Vietnam War (National Academies of Sciences, Engineering, and Medicine, 2018) and between American football and chronic traumatic encephalopathy in the late 2000s (Lindsley, 2017) (new act-consequence links). And, of course, the discovery of SARS-CoV-2/COVID-19 in late 2019 (Zheng, 2020) (new consequence).
} 
In a pioneering article, Karni and Vierø (2013) propose a model of belief revision under growing awareness called reverse Bayesianism. Reverse Bayesianism posits that as a person becomes aware of a new act, consequence, or act-consequence link, she revises her beliefs in a way that preserves the relative likelihoods of events in the original state space. More specifically, the model postulates that (i) in the case of a new act or consequence, probability mass shifts proportionally away from the states in the original state space to the new states in the expanded state space, and (ii) in the case of a new act-consequence link, null states in the original state space become non-null, and probability mass shifts proportionally away from the original non-null states to the previously null states that become possible.

The reverse Bayesian model has (at least) three notable features. First, Karni and Vierø (2013) provide an axiomatic foundation for the model. The key axioms are the "consistency" axioms, which essentially require that preferences conditional on the original state of awareness are not altered by growing awareness. ${ }^{2}$ Second, the model is built upon a well-known choice-theoretic framework, subjective expected utility theory. The upshot is a belief revision theory that mirrors the familiar process of Bayesian updating. This feature prompts Dominiak and Tserenjigmid (2021, p. 3) to describe the reverse Bayesian model as "elegant."

Third, reverse Bayesianism does not fully determine the revised probability distribution over the expanded state space. This is because reverse Bayesianism implies restrictions on the revised probabilities of events in the original state space, but not on the probabilities of new events in the expanded state space. To borrow a term from the econometrics literature, reverse Bayesianism partially identifies the revised probability distribution. Karni and Vierø (2013, p. 2805) highlight this feature in their concluding remarks: "The model presented in this article predicts that, as awareness grows and the state space expands, the relative likelihoods of events in the original state space remain unchanged. The model is silent about the absolute levels of these probabilities. In other words, our theory does not predict the probability of the new events in the expanded state space."

\footnotetext{
${ }^{2}$ Dominiak and Tserenjigmid (2018) show that the "invariant risk preferences" axiom is redundant given the consistency axioms and the SEU axioms.
} 
A natural question arises from this last feature: Under what conditions does reverse Bayesianism fully determine the revised probability distribution over the expanded state space? In this paper, we provide one such condition: act independence. Roughly speaking, act independence requires that the "act events" in the expanded state space are statistically independent. An act event is the collection of states in which a given act yields a given consequence. We show that under act independence, knowledge of the probabilities of the newly discovered act events is sufficient for reverse Bayesianism to fully determine the revised probability distribution in each case of growing awareness. This is our main set of results.

Act independence holds when acts are independent experiments or trials, akin to independent one-armed bandits. Whether act independence holds thus depends on the nature of the specific acts in question. For instance, whether fracking for natural gas results in groundwater contamination is likely to be independent of whether importing liquefied natural gas results in a fire or explosion. By contrast, whether one contracts HIV from sharing drug injection needles is likely to be related to whether one contracts HIV from having unprotected sex, since both depend on the prevalence of HIV in the population.

When act independence does not hold, a second question arises: What knowledge is sufficient for reverse Bayesianmism to fully determine the revised probability distribution? For any state in the expanded state space, knowledge of the joint probability of its "constituent" act events - i.e., the act events whose intersection defines such state - is plainly sufficient to pin down the probability of such state. In a second set of results, we derive implications of this fact in each case of growing awareness. In the case of a new act or act-consequence link, we show that reverse Bayesianism pins down the probability of any new state in the expanded state space with knowledge of (i) the probability of the newly discovered act event that contains such state and (ii) the correlation between such newly discovered act event and the intersection of the other constituent act events for such state. ${ }^{3}$ In the case of a

\footnotetext{
${ }^{3}$ This result - that knowledge of (i) and (ii) is a sufficient substitute for knowledge of the joint probability of the constituent act events - are reminiscient of the well-known result that knowledge of (a) the marginal distributions of a set of random variables and (b) the copula function that describes their dependence structure is a sufficient substitute for knowledge of the variables' joint distribution (Sklar, 1973).
} 
new consequence, the same knowledge is sufficient when the new consequence is linked to a single act, but not in general. In general, the decision maker would have to know (i) the joint probability of the newly discovered act events that contain the new state and (ii) the correlation between the intersection of such newly discovered act events and the intersection of the other constituent act events for such state.

Related literature. The unawareness literature was pioneered by Fagin and Halpern (1988). Other early contributions include Modica and Rustichini (1994, 1999), Dekel et al. (1998b), Halpern (2001), Heifetz et al. (2006), and Halpern and Rêgo (2008). The early papers in the literature generally pursued an epistemic approach or a game-theoretic approach. Surveys of these papers are provided by Schipper (2014b) (which offers a "gentle introduction" to the literature) and Schipper (2015) (which provides an extended review).

Karni and Vierø (2013) are among the pioneers of the choice-theoretic approach (i.e., the state-space approach) to modeling unawareness. Subsequent papers build on their approach. For instance, Grant et al. (2019) invoke their approach to model learning by experimentation in a world with unawareness; Karni and Vierø (2015, 2017) and Karni et al. (2021) extend the reverse Bayesian model to the cases where the decision maker is probabilistically sophisticated (but does not necessarily abide by expected utility theory), where she anticipates her growing awareness, and where the discovery of new consequences nullifies some states that were non-null before the discovery; and Dominiak and Tserenjigmid (2021) generalize the model such that the decision maker perceives ambiguity in the wake of growing awareness. ${ }^{4}$ Karni and Vierø (2013, 2017), Karni et al. (2021), and Dominiak and Tserenjigmid (2021) survey the papers that take a choice-theoretic approach. ${ }^{5}$

\footnotetext{
${ }^{4}$ More specifically, Dominiak and Tserenjigmid (2021) provide a theory of choice under growing awareness. They assume the decision maker originally has maxmin expected utility preferences (with unawareness) that are updated to posterior maxmin expected utility preferences (without unawareness). Moreover, they show that observing unexpected events may be a cause of ambiguity.

${ }^{5}$ In a recent working paper, Becker et al. (2021) report experimental evidence that is consistent with reverse Bayesianism. At the same time, Chambers and Hayashi (2018) criticize the model's empirical content from a revealed preference perspective. They show that, in the case of a new consequence, the model does not make singular predictions about observable choices over feasible acts.
} 
Structure of the paper. Section 2 presents the reverse Bayesian model. Section 3 introduces act independence and derives our main results. Section 4 contains our results on reserve Bayesianism without act independence. Section 5 offers brief concluding remarks. The Appendix collects the proofs of all theorems.

\section{Reverse Bayesian Model}

The primitives of the reverse Bayesian model are a finite, non-empty set $F$ of feasible acts and a finite, non-empty set $Z$ of feasible consequences. States are functions from the set of acts to the set of consequences. A state assigns a consequence to each act. The set of all possible states, $Z^{F}$, defines the conceivable state space. With $m$ acts and $n$ consequences, there are $n^{m}$ conceivable states.

The decision maker originally conceives the set of acts to be $F=\left\{f_{1}, \ldots, f_{m}\right\}$ and the set of consequences to be $Z=\left\{z_{1}, \ldots, z_{n}\right\}$. The conceivable state space is $Z^{F}=\left\{s_{1}, \ldots, s_{n^{m}}\right\}$, where each state $s=\left(s^{1}, \ldots, s^{m}\right) \in Z^{F}$ is a vector of length $m$, the $i$ th element of which, $s^{i}$, is the consequence $z_{j} \in Z$ produced by act $f_{i} \in F$ in that state of the world.

An act-consequence link, or link, is a causal relationship between an act and a consequence. The conceivable state space admits all conceivable links. However, the decision maker may perceive one or more links as infeasible, which brings her to nullify the states that admit such link. We refer to these as null states and denote them by $N \subset Z^{F}$. Taking only the non-null states defines the feasible state space, $S \equiv Z^{F} \backslash N$. There are $\prod_{i=1}^{m}\left(n-\nu_{i}\right)$ feasible states, where $\nu_{i}$ denotes the number of nullified links involving act $f_{i}$.

The decision maker's beliefs are represented by a probability measure $p$ on the conceivable state space, $Z^{F}$. The support of $p$ is the feasible state space, $S$. That is, $p(s)>0$ for all $s \in S$ and $p(s)=0$ for all $s \in N$.

The decision maker may initially fail to conceive one or more acts or consequences or to perceive as feasible one or more conceivable links. We refer to such failures of conception 
or perception as unawareness. However, the decision maker may later discover a new act or consequence, which expands both the feasible state space and the conceivable state space, or a new link, which expands the feasible state space but not the conceivable state space. ${ }^{6}$ We refer to such discoveries and expansions as growing awareness.

To illustrate, suppose $S=Z^{F}$ and the decision maker discovers a new consequence, $z_{n+1}$. Assuming the decision maker links the new consequence to every act, the set of consequences becomes $\widehat{Z}=Z \cup\left\{z_{n+1}\right\}$ and the feasible and conceivable state spaces both expand to $\widehat{S}=\widehat{Z}^{F}=\left\{s_{1}, \ldots, s_{(n+1)^{m}}\right\}$, where each state remains a vector of length $m$. Alternatively, suppose the decision maker discovers a new act, $f_{m+1}$. Then the set of acts becomes $\widehat{F}=$ $F \cup\left\{f_{m+1}\right\}$ and, assuming the decision maker links the new act to every consequence, the feasible and conceivable state spaces both expand to $\widehat{S}=Z^{\widehat{F}}=\left\{s_{1}, \ldots, s_{n^{(m+1)}}\right\}$, where each state now is a vector of length $m+1$. Lastly, suppose $S \subset Z^{F}$ because (and only because) the decision maker initially perceives as infeasible the link from $f_{1}$ to $z_{n}$. Discovery of the link from $f_{1}$ to $z_{n}$ does not alter the conceivable state space, but the feasible state space expands to coincide with the conceivable state space: $\widehat{S}=Z^{F}$. Section 3 contains illustrative depictions of conceivable and feasible state spaces and their expansion due to the discovery of new acts, consequences, and links.

In the wake of growing awareness, the decision maker revises her beliefs in a way that preserves the relative likelihoods of the events in the original feasible state space (the non-null events in the original conceivable state space). In each case of growing awareness, probability mass shifts proportionally away from the events in the original feasible state space to the new events in the expanded feasible state space. In the case of a new act or consequence, the new events in the expanded feasible state space are also new events in the expanded conceivable state space. In the case of a new link, the new events in the expanded feasible state space are the null events in the original conceivable state space that become non-null.

\footnotetext{
${ }^{6}$ To be clear, by "new" we mean "not previously conceived" in the case of acts and consequences, and "previously conceived but perceived as infeasible" in the case of links.
} 
Karni and Vierø (2013) refer to this belief revision process as reverse Bayesianism. Let $\widehat{p}$ denote the decision maker's revised beliefs on the expanded feasible state space, $\widehat{S}$. Formally, reverse Bayesianism implies two restrictions on $\widehat{p}$ : (i) in the case of a new consequence or link, $p(s) / p(t)=\widehat{p}(s) / \widehat{p}(t)$ for all $s, t \in S$; and (ii) in the case of a new act, $p(s) / p(t)=$ $\widehat{p}(E(s)) / \widehat{p}(E(t))$ for all $s, t \in S$, where $E(s)$ denotes the event in $\widehat{S}$ that corresponds to state $s \in S$; that is, given a new act $f_{m+1}, E(s) \equiv\left\{t \in \widehat{S}: t^{i}=s^{i}\right.$ for all $\left.i \neq m+1\right\}$.

\section{Act Independence}

We add an assumption to the reverse Bayesian model—act independence. Let $A_{i}\left(z_{j}\right) \subset \widehat{S}$ denote the event that act $f_{i}$ yields consequence $z_{j}$; that is, $A_{i}\left(z_{j}\right) \equiv\left\{t \in \widehat{S}: t^{i}=z_{j}\right\}$ is the collection of states in which act $f_{i}$ yields consequence $z_{j}$. We refer to events of this type as act events, and for each act event $A_{i}\left(z_{j}\right)$ we refer to the act $f_{i}$ as the predicate act. We make the following assumption about the act events in $\widehat{S}$.

Act independence. For every collection of act events in $\widehat{S}$ such that no two act events have the same predicate act, the act events in the collection are mutually independent.

Act independence implies additional restrictions on the decision maker's revised beliefs, $\widehat{p}$. Observe that we can express each state $s=\left(s^{1}, \ldots, s^{m}\right) \in \widehat{S}$ as the intersection of a unique collection of act events in $\widehat{S}: s=\bigcap_{i} A_{i}\left(s^{i}\right)$. We refer to this collection as the constituent act events for state $s$. Act independence implies that the probability of state $s$ equals the product of the probabilities of its constituent act events: $\widehat{p}(s)=\prod_{i} \widehat{p}\left(A_{i}\left(s^{i}\right)\right)$ for all $s \in \widehat{S}$.

Growing awareness - whether it entails a new act, consequence, or link - gives rise to one or more new act events in $\widehat{S}$. In the remainder of this section we show that, under act independence, knowledge of the probabilities of the new act events in $\widehat{S}$ is sufficient to fully determine $\widehat{p}$ in each case of growing awareness. We start with the case of a new link. 


\subsection{New Link}

Suppose $S \subset Z^{F}$ and the decision maker discovers a new link from $f_{l}$ to $z_{k}$ for some $l \in$ $\{1, \ldots, m\}$ and $k \in\{1, \ldots, n\}$. Let $\widehat{S}$ denote the expanded feasible state space and $\widehat{p}$ denote the decision maker's revised beliefs on $\widehat{S}$. Observe that $\widehat{S}=S \cup \Delta$, where $\Delta=A_{l}\left(z_{k}\right)$ is the newly discovered event that $f_{l}$ yields $z_{k}$. Intuitively, $\Delta$ is a copy of any one of the act events $A_{l}\left(z_{j}\right)$ in $S$, except that $f_{l}$ yields $z_{k}$ (instead of $\left.z_{j}\right)$ in every state in $\Delta$. We assume that, by virtue of the discovery, the decision maker learns that $f_{l}$ yields $z_{k}$ with probability $\delta>0$. By defintion, $\delta=\widehat{p}(\Delta)$.

For each state $s \in \Delta$, let $L(s) \equiv\left\{t \in S: t^{i}=s^{i}, \forall i \neq l\right\}$ denote the event in $S$ that corresponds to the state $s \in \Delta$. In other words, $L(s)$ comprises the states in $S$ in which every act (other than $f_{l}$ ) yields the same consequence that it yields in state $s \in \Delta$.

By reverse Bayesianism, the relative likelihoods of the states in $S$ are preserved: $p(s) / p(t)=$ $\widehat{p}(s) / \widehat{p}(t)$ for all $s, t \in S$. By act independence, the probability of each state in $\Delta$ equals the product of the probabilities of its constituent act events in $\widehat{S}$ - that is, $\widehat{p}(s)=\prod_{i=1}^{m} \widehat{p}\left(A_{i}\left(s^{i}\right)\right)$ for all $s=\left(s^{1}, \ldots, s^{m}\right) \in \Delta$. It follows that:

Theorem 1. In the case of a new link involving $f_{l}$ :

(i) $\widehat{p}(s)=(1-\delta) p(s)$ for all $s \in S$; and

(ii) $\widehat{p}(s)=\delta p(L(s))$ for all $s \in \Delta$.

Theorem 1 says that (i) the fraction $\delta$ of the probability mass of each state in $S$ is taken away, and that (ii) the total probability mass $\delta$ taken away from the states in $S$ is distributed among the states in $\Delta$ in proportion to the probability masses of their corresponding events in $S$. Reverse Bayesianism dictates the first result (how probability mass is shifted away from the states in $S$ ), while act independence dictates the second result (how the shifted probability mass is apportioned among the states in $\Delta$ ). Together, reverse Bayesianism and act independence fully determine the revised probability distribution $\widehat{p}$ on $\widehat{S}{ }^{7}$

\footnotetext{
${ }^{7}$ Note that $p$ is the Bayesian update of $\widehat{p}$ conditional on the event $S$; hence the term reverse Bayesianism.
} 
Example 1. Consider the $2 \times 2$ case: $F=\left\{f_{1}, f_{2}\right\}$ and $Z=\left\{z_{1}, z_{2}\right\}$; the conceivable state space, $Z^{F}$, comprises four states, $s_{1}=\left(z_{1}, z_{1}\right), s_{2}=\left(z_{1}, z_{2}\right), s_{3}=\left(z_{2}, z_{1}\right)$, and $s_{4}=\left(z_{2}, z_{2}\right)$; and the decision maker's initial beliefs are $p=\left(p_{1}, \ldots, p_{4}\right)$ where $p_{k} \equiv p\left(s_{k}\right)$ for all $k$.

Suppose the decision maker initially fails to conceive that act $f_{1}$ can yield consequence $z_{2}$. That is, suppose she initially perceives the event $\Delta=A_{1}\left(z_{2}\right)=\left\{s_{3}, s_{4}\right\}$ as infeasible (null). This implies $p_{3}=p_{4}=0$. We can depict the original feasible state space, $S \subset Z^{F}$, and the decision maker's initial beliefs, $p$, as follows:

\begin{tabular}{c|cc}
$p$ & $p_{1}$ & $p_{2}$ \\
\hline \hline$F \backslash S$ & $s_{1}$ & $s_{2}$ \\
\hline$f_{1}$ & $z_{1}$ & $z_{1}$ \\
$f_{2}$ & $z_{1}$ & $z_{2}$.
\end{tabular}

Suppose the decision maker subsequently discovers that $f_{1}$ can yield $z_{2}$. The feasible state space expands to $\widehat{S}=S \cup \Delta$ and the decision maker revises her beliefs from $p$ to $\widehat{p}$ :

\begin{tabular}{c|cccc}
$\widehat{p}$ & $\widehat{p}_{1}$ & $\widehat{p}_{2}$ & $\widehat{p}_{3}$ & $\widehat{p}_{4}$ \\
\hline \hline$F \backslash \widehat{S}$ & $s_{1}$ & $s_{2}$ & $s_{3}$ & $s_{4}$ \\
\hline$f_{1}$ & $z_{1}$ & $z_{1}$ & $z_{2}$ & $z_{2}$ \\
$f_{2}$ & $z_{1}$ & $z_{2}$ & $z_{1}$ & $z_{2}$
\end{tabular}

Observe that for each state $s$ in $\Delta$ there is an event $L(s)$ in $S$ that corresponds with $s$ on $f_{2}$. Specifically, $L\left(s_{3}\right)=\left\{s_{1}\right\}$ and $L\left(s_{4}\right)=\left\{s_{2}\right\}$. We assume that, by virtue of the discovery, the decision maker learns that $f_{1}$ yields $z_{2}$ with probability $\delta=\widehat{p}(\Delta)=\widehat{p}_{3}+\widehat{p}_{4}$. Reverse Bayesiansim implies $\frac{p_{1}}{p_{2}}=\frac{\widehat{p}_{1}}{\widehat{p}_{2}}$, and act independence implies $\frac{\widehat{p}_{1}}{\widehat{p}_{2}}=\frac{\widehat{p}_{3}}{\widehat{p}_{4}}$. It follows that $\widehat{p}$ is given by $\widehat{p}_{1}=(1-\delta) p_{1}, \widehat{p}_{2}=(1-\delta) p_{2}, \widehat{p}_{3}=\delta p_{1}$, and $\widehat{p}_{4}=\delta p_{2}$. 


\subsection{New Act}

Next, suppose $S \subseteq Z^{F}$ and the decision maker discovers a new act, $f_{m+1}$. Again, let $\widehat{S}$ denote the expanded feasible state space and $\widehat{p}$ denote the decision maker's revised beliefs on $\widehat{S}$. Observe that $\widehat{S}=\bigcup_{j=1}^{n} \Delta_{j}$, where $\Delta_{j}=A_{m+1}\left(z_{j}\right)$ is the newly discovered event that $f_{m+1}$ yields $z_{j}$. Intuitively, each $\Delta_{j}$ is an augmented copy of $S$ in which $f_{m+1}$ yields $z_{j}$ in every state. We assume that, by virtue of the discovery, the decision maker learns that $f_{m+1}$ yields $z_{j}$ with probability $\delta_{j}>0$ for all $j=1, \ldots, n .{ }^{8}$ Note that $\delta_{j}=\widehat{p}\left(\Delta_{j}\right)$ and $\sum_{j=1}^{n} \delta_{j}=1$.

For each state $s \in S$, let $E(s) \equiv\left\{t \in \widehat{S}: t^{i}=s^{i}, \forall i \neq m+1\right\}$ denote the event in $\widehat{S}$ that corresponds to the state $s \in S$. In other words, $E(s)$ comprises the states in $\widehat{S}$ in which every act (other than $f_{m+1}$ ) yields the same consequence that it yields in state $s \in S$. Observe that $\widehat{S}=\bigcup_{s \in S} E(s)$, where $E(s)$ comprises $n$ states, one in which $f_{m+1}$ yields $z_{1}$, one in which $f_{m+1}$ yields $z_{2}$, and so forth. Index the states in each $E(s)$ by $j=1, \ldots, n$, such that $s_{j} \in E(s)$ is the state in $E(s)$ in which $f_{m+1}$ yields $z_{j}$. The connection between the sets of events $\{E(s): s \in S\}$ and $\left\{\Delta_{j}: j=1, \ldots, n\right\}$, both of which partition $\widehat{S}$, is that $\Delta_{j}$ collects the $j$ th state from each $E(s)$.

By reverse Bayesianism, $p(s) / p(t)=\widehat{p}(E(s)) / \widehat{p}(E(t))$ for all $s, t \in S$. By act independence, $\widehat{p}(s)=\prod_{i=1}^{m+1} \widehat{p}\left(A_{i}\left(s^{i}\right)\right)$ for all $s=\left(s^{1}, \ldots, s^{m+1}\right) \in \widehat{S}$. It follows that:

Theorem 2. In the case of a new act $f_{m+1}$, for all $s \in S$ and corresponding $E(s) \subset \widehat{S}$, $\widehat{p}\left(s_{j}\right)=\delta_{j} p(s)$ for all $s_{j} \in E(s), j=1, \ldots, n$.

Here is the intuition behind Theorem 2. After the discovery of $f_{m+1}$, each state $s \in S$ is split into $n$ states $s_{j} \in \widehat{S}$, one for each consequence $z_{j}, j=1, \ldots, n$. (In state $s_{j}, f_{m+1}$ yields $z_{j}$.) These $n$ states comprise the event $E(s) \subset \widehat{S}$ that corresponds to the state $s \in S$. For each state $s \in S$, reverse Bayesianism dictates that its probability mass is shifted to the corresponding event $E(s) \subset \widehat{S}$, while act independence dictates that the fraction $\delta_{j}$ of the shifted probability mass is apportioned to state $s_{j} \in E(s)$ for all $j=1, \ldots, n$.

\footnotetext{
${ }^{8}$ Assuming $\delta_{j}>0$ for all $j=1, \ldots, n$ is without loss of generality. We can deal with the case where $\delta_{j}=0$ for some $j$ by assuming $\delta_{j}>0$ for the first $k<n$ and changing $n$ to $k$ as necessary in the statements below.
} 
Example 2. Consider the $2 \times 2$ case and assume the original feasible state space is $S=Z^{F}$ :

\begin{tabular}{c|cccc}
$p$ & $p_{1}$ & $p_{2}$ & $p_{3}$ & $p_{4}$ \\
\hline \hline$F \backslash S$ & $s_{1}$ & $s_{2}$ & $s_{3}$ & $s_{4}$ \\
\hline$f_{1}$ & $z_{1}$ & $z_{1}$ & $z_{2}$ & $z_{2}$ \\
$f_{2}$ & $z_{1}$ & $z_{2}$ & $z_{1}$ & $z_{2}$
\end{tabular}.

Suppose the decision maker discovers a new act $f_{3}$ which she perceives can yield $z_{1}$ or $z_{2}$. The expanded feasible state space is $\widehat{S}=\Delta_{1} \cup \Delta_{2}$, where $\Delta_{1}=A_{3}\left(z_{1}\right)=\left\{s_{1}, s_{2}, s_{3}, s_{4}\right\}$ and $\Delta_{2}=A_{3}\left(z_{2}\right)=\left\{s_{5}, s_{6}, s_{7}, s_{8}\right\}:$

\begin{tabular}{c|cccccccc}
$\widehat{p}$ & $\widehat{p}_{1}$ & $\widehat{p}_{2}$ & $\widehat{p}_{3}$ & $\widehat{p}_{4}$ & $\widehat{p}_{5}$ & $\widehat{p}_{6}$ & $\widehat{p}_{7}$ & $\widehat{p}_{8}$ \\
\hline \hline$F \backslash \widehat{S}$ & $s_{1}$ & $s_{2}$ & $s_{3}$ & $s_{4}$ & $s_{5}$ & $s_{6}$ & $s_{7}$ & $s_{8}$ \\
\hline$f_{1}$ & $z_{1}$ & $z_{1}$ & $z_{2}$ & $z_{2}$ & $z_{1}$ & $z_{1}$ & $z_{2}$ & $z_{2}$ \\
$f_{2}$ & $z_{1}$ & $z_{2}$ & $z_{1}$ & $z_{2}$ & $z_{1}$ & $z_{2}$ & $z_{1}$ & $z_{2}$ \\
$f_{3}$ & $z_{1}$ & $z_{1}$ & $z_{1}$ & $z_{1}$ & $z_{2}$ & $z_{2}$ & $z_{2}$ & $z_{2}$
\end{tabular}.

Observe that $\Delta_{1}$ is an augmented copy of $S$ in which $f_{3}$ yields $z_{1}$ in every state, and that $\Delta_{2}$ is an augmented copy of $S$ in which $f_{3}$ yields $z_{2}$ in every state. Stated differently, each state in $S$ is split into two depending on whether $f_{3}$ yields $z_{1}$ or $z_{2}$. Hence, for each state $s$ in $S$ there is a corresponding event $E(s)$ in $\widehat{S}$. Specifically, $E\left(s_{1}\right)=\left\{s_{1}, s_{5}\right\}, E\left(s_{2}\right)=\left\{s_{2}, s_{6}\right\}, E\left(s_{3}\right)=$ $\left\{s_{3}, s_{7}\right\}$, and $E\left(s_{4}\right)=\left\{s_{4}, s_{8}\right\}$. We assume that, by virtue of the discovery, the decision maker learns that $f_{3}$ yields $z_{2}$ with probability $\delta$. Thus, $1-\delta=\widehat{p}\left(\Delta_{1}\right)=\widehat{p}_{1}+\widehat{p}_{2}+\widehat{p}_{3}+\widehat{p}_{4}$ and $\delta=\widehat{p}\left(\Delta_{2}\right)=\widehat{p}_{5}+\widehat{p}_{6}+\widehat{p}_{7}+\widehat{p}_{8}$. Reverse Bayesiansim implies $\frac{p_{1}}{p_{2}}=\frac{\widehat{p}_{1}+\widehat{p}_{5}}{\widehat{p}_{2}+\widehat{p}_{6}}, \frac{p_{2}}{p_{3}}=\frac{\widehat{p}_{2}+\widehat{p}_{6}}{\widehat{p}_{3}+\widehat{p}_{7}}$, and $\frac{p_{3}}{p_{4}}=\frac{\widehat{p}_{3}+\widehat{p}_{7}}{\widehat{p}_{4}+\widehat{p}_{8}}$, and act independence implies $\frac{\widehat{p}_{1}}{\widehat{p}_{2}}=\frac{\widehat{p}_{5}}{\widehat{p}_{6}}, \frac{\widehat{p}_{2}}{\widehat{p}_{3}}=\frac{\widehat{p}_{6}}{\widehat{p}_{7}}$, and $\frac{\widehat{p}_{3}}{\widehat{p}_{4}}=\frac{\widehat{p}_{7}}{\widehat{p}_{8}}$. It follows that the revised probability distribution $\widehat{p}$ is given by $\widehat{p}_{1}=(1-\delta) p_{1}, \widehat{p}_{2}=(1-\delta) p_{2} \widehat{p}_{3}=(1-\delta) p_{3}$, $\widehat{p}_{4}=(1-\delta) p_{4}, \widehat{p}_{5}=\delta p_{1}, \widehat{p}_{6}=\delta p_{2}, \widehat{p}_{7}=\delta p_{3}$, and $\widehat{p}_{8}=\delta p_{4}$. 


\subsection{New Consequence}

Last, suppose $S \subseteq Z^{F}$ and the decision maker discovers a new consequence, $z_{n+1}$. Once again, let $\widehat{S}$ denote the expanded feasible state space and $\widehat{p}$ denote the decision maker's revised beliefs on $\widehat{S}$. Observe that $\widehat{S}=S \cup \Delta$, where $\Delta=\bigcup_{i=1}^{m} A_{i}\left(z_{n+1}\right)$ is the union of the newly discovered events that $f_{i}$ yields $z_{n+1}$ for all $i=1, \ldots, m$. We assume that, by virtue of the discovery, the decision maker learns that $f_{i}$ yields $z_{n+1}$ with probability $\alpha_{i}>0$ for all $i=1, \ldots, m .^{9}$ That is, $\alpha_{i}=\widehat{p}\left(A_{i}\left(z_{n+1}\right)\right)$. Let $\delta=\widehat{p}(\Delta)$ and note that $1-\delta=\prod_{i=1}^{m}\left(1-\alpha_{i}\right)$.

For each state $s \in \Delta$, let $I(s) \equiv\left\{i \in\{1, \ldots, m\}: s^{i}=z_{n+1}\right\}$ denote the indices of the acts that yield $z_{n+1}$ in that state of the world, and let $\bar{I}(s) \equiv\left\{i \in\{1, \ldots, m\}: s^{i} \neq z_{n+1}\right\}$ denote the indices of the acts that do not yield $z_{n+1}$ in that state of the world. In addition, for each $s \in \Delta$, let $C(s) \equiv\left\{t \in S: t^{i}=s^{i}, \forall i \in \bar{I}(s)\right\}$ denote the event in $S$ that corresponds to $s \in \Delta$ on $\bar{I}(s)$. In other words, $C(s)$ comprises the states in $S$ in which every act (other than the acts that yield $z_{n+1}$ ) yields the same consequence that it yields in state $s \in \Delta$.

By reverse Bayesianism, $p(s) / p(t)=\widehat{p}(s) / \widehat{p}(t)$ for all $s, t \in S$. By act independence, $\widehat{p}(s)=\prod_{i=1}^{m} \widehat{p}\left(A_{i}\left(s^{i}\right)\right)$ for all $s=\left(s^{1}, \ldots, s^{m}\right) \in \Delta$. It follows that:

Theorem 3. In the case of a new consequence $z_{n+1}$ :

(i) $\widehat{p}(s)=\left(\prod_{i=1}^{m}\left(1-\alpha_{i}\right)\right) p(s)$ for all $s \in S$;

(ii) $\widehat{p}(s)=\left(\prod_{i \in I(s)} \alpha_{i}\right)\left(\prod_{i \in \bar{I}(s)}\left(1-\alpha_{i}\right)\right) p(C(s))$ for all $s \in \Delta$ such that $I(s) \subset\{1, \ldots, m\}$;

(iii) $\widehat{p}(s)=\prod_{i=1}^{m} \alpha_{i}$ for the $s \in \Delta$ such that $I(s)=\{1, \ldots, m\}$.

Theorem 3 is similar to Theorem 1 . The first result says that the fraction $\delta$ of the probability mass of each state in $S$ is taken away. (Recall that $1-\delta=\prod_{i=1}^{m}\left(1-\alpha_{i}\right)$.) This result is dictated by reverse Bayesianism. The second and third results say how the total probability mass $\delta$ taken away from the states in $S$ is distributed among the states in $\Delta$. These results are dictated by act independence. Specifically, the third results says that probability mass $\prod_{i=1}^{m} \alpha_{i}$ is apportioned to the one state in which every act results

\footnotetext{
${ }^{9}$ Assuming $\alpha_{i}>0$ for all $i$ is without loss of generality. We can deal with the case where $\alpha_{i}=0$ for some $i$ by assuming $\alpha_{i}>0$ for the first $l<m$ and changing $m$ to $l$ as necessary in the statements below.
} 
in $z_{n+1}$ (this is a clear implication of act independence), while the second result says that the remaining probability mass, $\delta-\prod_{i=1}^{m} \alpha_{i}$, is distributed among the other states in $\Delta$ in proportion to the probability masses of their corresponding events in $S$.

Example 3. Consider the $2 \times 2$ case and assume the original feasible state space is $S=Z^{F}$. Suppose the decision maker discovers a new consequence $z_{3}$ which she links to $f_{1}$ and $f_{2}$. The expanded feasible state space is $\widehat{S}=S \cup \Delta$, where $\Delta=A_{1}\left(z_{3}\right) \cup A_{2}\left(z_{3}\right)=\left\{s_{5}, s_{6}, s_{7}, s_{8}, s_{9}\right\}$ :

\begin{tabular}{c|ccccccccc}
$\widehat{p}$ & $\widehat{p}_{1}$ & $\widehat{p}_{2}$ & $\widehat{p}_{3}$ & $\widehat{p}_{4}$ & $\widehat{p}_{5}$ & $\widehat{p}_{6}$ & $\widehat{p}_{7}$ & $\widehat{p}_{8}$ & $\widehat{p}_{9}$ \\
\hline \hline$F \backslash \widehat{S}$ & $s_{1}$ & $s_{2}$ & $s_{3}$ & $s_{4}$ & $s_{5}$ & $s_{6}$ & $s_{7}$ & $s_{8}$ & $s_{9}$ \\
\hline$f_{1}$ & $z_{1}$ & $z_{1}$ & $z_{2}$ & $z_{2}$ & $z_{3}$ & $z_{3}$ & $z_{1}$ & $z_{2}$ & $z_{3}$ \\
$f_{2}$ & $z_{1}$ & $z_{2}$ & $z_{1}$ & $z_{2}$ & $z_{1}$ & $z_{2}$ & $z_{3}$ & $z_{3}$ & $z_{3}$
\end{tabular}.

Observe that for each state $s$ in $\Delta$ there is an event $C(s)$ in $S$ that corresponds with $s$ on the act that does not yield $z_{3}$. Specifically, $C\left(s_{5}\right)=\left\{s_{1}, s_{3}\right\}, C\left(s_{6}\right)=\left\{s_{2}, s_{4}\right\}, C\left(s_{7}\right)=\left\{s_{1}, s_{2}\right\}$, $C\left(s_{8}\right)=\left\{s_{3}, s_{4}\right\}$, and $C\left(s_{9}\right)=\{\emptyset\} .{ }^{10}$ We assume that, by virtue of the discovery, the decision maker learns that $f_{1}$ yields $z_{3}$ with probability $\alpha$ and that $f_{2}$ yields $z_{3}$ with probability $\beta$. By defnition, $\alpha=\widehat{p}\left(A_{1}\left(z_{3}\right)\right)=\widehat{p}_{5}+\widehat{p}_{6}+\widehat{p}_{9}$ and $\beta=\widehat{p}\left(A_{2}\left(z_{3}\right)\right)=\widehat{p}_{7}+\widehat{p}_{8}+\widehat{p}_{9}$. It follows from Theorem 3 that the revised probability distribution $\widehat{p}$ is given by:

$$
\begin{aligned}
& \widehat{p}_{1}=(1-\alpha)(1-\beta) p_{1}, \quad \widehat{p}_{2}=(1-\alpha)(1-\beta) p_{2}, \quad \widehat{p}_{3}=(1-\alpha)(1-\beta) p_{3}, \\
& \widehat{p}_{4}=(1-\alpha)(1-\beta) p_{4}, \quad \widehat{p}_{5}=\alpha(1-\beta)\left(p_{1}+p_{3}\right), \quad \widehat{p}_{6}=\alpha(1-\beta)\left(p_{2}+p_{4}\right), \\
& \widehat{p}_{7}=\beta(1-\alpha)\left(p_{1}+p_{2}\right), \quad \widehat{p}_{8}=\beta(1-\alpha)\left(p_{3}+p_{4}\right), \quad \text { and } \quad \widehat{p}_{9}=\alpha \beta .
\end{aligned}
$$

\footnotetext{
${ }^{10}$ In this example, $I\left(s_{5}\right)=I\left(s_{6}\right)=\{1\}, I\left(s_{7}\right)=I\left(s_{8}\right)=\{2\}$, and $I\left(s_{9}\right)=\{1,2\}$. Accordingly, $\bar{I}\left(s_{5}\right)=$ $\bar{I}\left(s_{6}\right)=\{2\}, \bar{I}\left(s_{7}\right)=\bar{I}\left(s_{8}\right)=\{1\}$, and $\bar{I}\left(s_{9}\right)=\{\emptyset\}$.
} 
Let $\delta=\widehat{p}(\Delta)=\widehat{p}_{5}+\widehat{p}_{6}+\widehat{p}_{7}+\widehat{p}_{8}+\widehat{p}_{9}$. Note that $\delta=\alpha+\beta-\alpha \beta$ and $1-\delta=(1-\alpha)(1-\beta)$.

We can rewrite $\widehat{p}$ in terms of $\delta$ as follows:

$$
\begin{aligned}
& \widehat{p}_{1}=(1-\delta) p_{1}, \quad \widehat{p}_{2}=(1-\delta) p_{2}, \quad \widehat{p}_{3}=(1-\delta) p_{3}, \quad \widehat{p}_{4}=(1-\delta) p_{4}, \\
& \widehat{p}_{5}=(\delta-\beta)\left(p_{1}+p_{3}\right), \quad \widehat{p}_{6}=(\delta-\beta)\left(p_{2}+p_{4}\right), \quad \widehat{p}_{7}=(\delta-\alpha)\left(p_{1}+p_{2}\right), \\
& \widehat{p}_{8}=(\delta-\alpha)\left(p_{3}+p_{4}\right), \quad \text { and } \quad \widehat{p}_{9}=\alpha+\beta-\delta .
\end{aligned}
$$

\section{Reverse Bayesianism without Act Independence}

Of course, act independence does not always hold. When acts are not independent trials, act events with different predicate acts may be correlated. When this is the case, for any state in the expanded feasible state space, knowledge of the joint probability of such state's constituent act events is sufficient for reverse Bayesianism to pin down the probability of

such state. To see this, observe that $\widehat{p}(s)=\widehat{p}\left(\bigcap_{i} A_{i}\left(s^{i}\right)\right)$ for all $s \in \widehat{S}$. In this section, we derive implications of this fact for each case of growing awareness. In the case of a new act or new link, we show that reverse Bayesianism pins down the probability of any new state in the expanded feasible state space with knowledge of (i) the probability of the newly discovered act event that contains such state and (ii) the correlation between such newly discovered act event and the intersection of the other constituent act events for such state. In the case of a new consequence, the same knowledge is sufficient when the new consequence is linked to a single act, but not in general. In general, the decision maker would have to know (i) the joint probability of the newly discovered act events that contain the new state and (ii) the correlation between the intersection of such newly discovered act events and the intersection of the other constituent act events for such state. We start with the case of a new act. 


\subsection{New Act}

Suppose $S \subseteq Z^{F}$ and the decision maker discovers a new act, $f_{m+1}$. Let $\widehat{S}=\bigcup_{j=1}^{n} \Delta_{j}$ denote the expanded feasible state space, where $\Delta_{j}=A_{m+1}\left(z_{j}\right)$ is the newly discovered event that $f_{m+1}$ yields $z_{j}$, and let $\widehat{p}$ denote the decision maker's revised beliefs on $\widehat{S}$. We assume that, by virtue of the discovery, the decision maker learns $\delta_{j}=\widehat{p}\left(\Delta_{j}\right)$ for all $j$. As before, let $E(s)$ denote the event in $\widehat{S}$ that corresponds to the state $s \in S$. Observe that $E(s)=\bigcap_{i=1}^{m} A_{i}\left(s_{j}^{i}\right)$.

Theorem 4. Assume reverse Bayesianism. Take any $s \in S$ and corresponding $E(s) \subset \widehat{S}$. Take any $s_{j} \in E(s)$. Observe that $s_{j} \in \Delta_{j}$. The probability of $s_{j}$ is given by

$$
\widehat{p}\left(s_{j}\right)=\delta_{j} p(s)+\rho\left(\Delta_{j}, E(s)\right) \sqrt{\delta_{j}\left(1-\delta_{j}\right) p(s)(1-p(s))},
$$

where $\rho\left(\Delta_{j}, E(s)\right)$ denotes the correlation between $\Delta_{j}$ and $E(s)$.

Thus, knowledge of $\delta_{j}$ and $\rho\left(\Delta_{j}, E(s)\right)$ pins down $\widehat{p}\left(s_{j}\right)$. Note that $\widehat{p}\left(s_{j}\right)$ is equal to the value with act independence, $\delta_{j} p(s)$, plus a term to account for the correlation between $\Delta_{j}$ (the newly discovered act event that contains $s_{j}$ ) and $E(s)$ (the intersection of the other constituent act events for $\left.s_{j}\right){ }^{11}$

\subsection{New Link}

Next, suppose $S \subset Z^{F}$ and the decision maker discovers a new link from $f_{l}$ to $z_{k}$ for some $l \in\{1, \ldots, m\}$ and $k \in\{1, \ldots, n\}$. Let $\widehat{S}=S \cup \Delta$ denote the expanded feasible state space, where $\Delta=A_{l}\left(z_{k}\right)$ is the newly discovered event that $f_{l}$ yields $z_{k}$, and let $\widehat{p}$ denote the decision maker's revised beliefs on $\widehat{S}$. We assume that, by virtue of the discovery, the decision maker learns $\delta=\widehat{p}(\Delta)$. As before, let $L(s)$ denote the event in $S$ that corresponds to the state $s \in \Delta$. Observe that $L(s) \cup\{s\}=\bigcap_{i \neq l} A_{i}\left(s^{i}\right)$.

\footnotetext{
${ }^{11}$ Note that $s_{j}=\bigcap_{i=1}^{m+1} A_{i}\left(s_{j}^{i}\right)=A_{m+1}\left(z_{j}\right) \cap\left(\bigcap_{i=1}^{m} A_{i}\left(s_{j}^{i}\right)\right)=\Delta_{j} \cap E(s)$.
} 
Theorem 5. Assume reserve Bayesianism. Take any $s \in \Delta$. Let $y=p(L(s))$ and let $\rho=\rho(\Delta, L(s) \cup\{s\})$ denote the correlation between $\Delta$ and $L(s) \cup\{s\}$. Then

$$
\begin{aligned}
& \widehat{p}(s)=r_{1}=\delta y+\frac{\delta \rho^{2}}{2\left(1-\delta+\delta \rho^{2}\right)}\left[1-2 y+\sqrt{1+\left(4 y(1-\delta)(1-y) / \delta \rho^{2}\right)}\right], \text { if } \rho>0 \\
& \widehat{p}(s)=r_{2}=\delta y+\frac{\delta \rho^{2}}{2\left(1-\delta+\delta \rho^{2}\right)}\left[1-2 y-\sqrt{1+\left(4 y(1-\delta)(1-y) / \delta \rho^{2}\right)}\right], \text { if } \rho<0 .
\end{aligned}
$$

The value of $\widehat{p}(s)$ is determined by the roots of a quadratic equation. Because the correlation $\rho$ enters the coefficients of the quadratic equation and the expressions for its roots only through terms involving $\rho^{2}$, the quadratic equation and its roots are the same whether $\rho$ is positive or negative. In the proof of Theorem 5 , we derive the quadratic equation and show that its roots are given by $r_{1}$ and $r_{2}$. We further show that $r_{1}>\delta y>r_{2}$, and that $r_{1}$ applies for positive correlation and $r_{2}$ applies for negative correlation.

\subsection{New Consequence}

The results in the previous cases rely on the fact that:

(i) In the case of a new act, for $s_{j} \in \Delta_{j}$,

$$
\begin{aligned}
\widehat{p}\left(s_{j}\right) & =\widehat{p}\left(\bigcap_{i=1}^{m+1} A_{i}\left(s^{i}\right)\right)=\widehat{p}\left(A_{m+1}\left(z_{j}\right) \cap\left(\bigcap_{i=1}^{m} A_{i}\left(s_{j}^{i}\right)\right)\right)=\widehat{p}\left(\Delta_{j} \cap E(s)\right) \\
& =\widehat{p}\left(\Delta_{j}\right) \widehat{p}(E(s))+\rho\left(\Delta_{j}, E(s)\right) \sqrt{\widehat{p}\left(\Delta_{j}\right)\left(1-\widehat{p}\left(\Delta_{j}\right)\right) \widehat{p}(E(s))(1-\widehat{p}(E(s)))}
\end{aligned}
$$

(ii) In the case of a new link, for $s \in \Delta$,

$$
\begin{aligned}
\widehat{p}(s)= & \widehat{p}\left(\bigcap_{i=1}^{m} A_{i}\left(s^{i}\right)\right)=\widehat{p}\left(A_{l}\left(z_{k}\right) \cap\left(\bigcap_{i \neq l} A_{i}\left(s^{i}\right)\right)\right)=\widehat{p}(\Delta \cap(L(s) \cup\{s\})) \\
= & \widehat{p}(\Delta) \widehat{p}(L(s) \cup\{s\}) \\
& \quad+\rho(\Delta, L(s) \cup\{s\}) \sqrt{\widehat{p}(\Delta)(1-\widehat{p}(\Delta)) \widehat{p}(L(s) \cup\{s\})(1-\widehat{p}(L(s) \cup\{s\}))} .
\end{aligned}
$$


From these equations we can see that knowledge of (i) $\delta_{j}=\widehat{p}\left(\Delta_{j}\right)$ or $\delta=\widehat{p}(\Delta)$, as the case may be, and (ii) $\rho\left(\Delta_{j}, E(s)\right)$ or $\rho(\Delta, L(s) \cup\{s\})$, as the case may be, is a sufficient substitute for knowledge of $\widehat{p}\left(\bigcap_{i} A_{i}\left(s^{i}\right)\right) .{ }^{12}$

In general, however, the case of a new consequence is different. Suppose $S \subseteq Z^{F}$ and the decision maker discovers a new consequence, $z_{n+1}$. Let $\widehat{S}=S \cup \Delta$ denote the expanded feasible state space, where $\Delta=\bigcup_{i=1}^{m} A_{i}\left(z_{n+1}\right)$ is the union of the newly discovered events that $f_{i}$ yields $z_{n+1}$ for all $i=1, \ldots, m$, and let $\widehat{p}$ denote the decision maker's revised beliefs on $\widehat{S}$. We assume that, by virtue of the discovery, the decision maker learns $\alpha_{i}=\widehat{p}\left(A_{i}\left(z_{n+1}\right)\right)$ for all $i=1, \ldots, m$. As before, let $I(s)$ denote the indices of the acts that yield $z_{n+1}$ in state $s \in \Delta$, let $\bar{I}(s)$ denote the indices of the acts that do not yield $z_{n+1}$ in state $s \in \Delta$, and let $C(s)$ denote the event in $S$ that corresponds to state $s \in \Delta$ on $\bar{I}(s)$.

Let $\mathcal{A}=\bigcap_{i \in I(s)} A_{i}\left(s^{i}\right)$ and observe that $C(s) \cup\{s\}=\bigcap_{i \in \bar{I}(s)} A_{i}\left(s^{i}\right)$. Thus, in the case of a new consequence, for $s \in \Delta$,

$$
\begin{aligned}
\widehat{p}(s)= & \widehat{p}\left(\bigcap_{i=1}^{m} A_{i}\left(s^{i}\right)\right)=\widehat{p}\left(\left(\bigcap_{i \in I(s)} A_{i}\left(s^{i}\right)\right) \cap\left(\bigcap_{i \in \bar{I}(s)} A_{i}\left(s^{i}\right)\right)\right)=\widehat{p}(\mathcal{A} \cap(C(s) \cup\{s\})) \\
= & \widehat{p}(\mathcal{A}) \widehat{p}(C(s) \cup\{s\}) \\
& +\rho(\mathcal{A}, C(s) \cup\{s\}) \sqrt{\widehat{p}(\mathcal{A})(1-\widehat{p}(\mathcal{A})) \widehat{p}(C(s) \cup\{s\})(1-\widehat{p}(C(s) \cup\{s\}))} .
\end{aligned}
$$

From this equation we can see that knowledge of (i) $\widehat{p}(\mathcal{A})$ and (ii) $\rho(\mathcal{A}, C(s) \cup\{s\})$ is a sufficient substitute for knowledge of $\widehat{p}\left(\bigcap_{i} A_{i}\left(s^{i}\right)\right) \cdot{ }^{13}$ But, in general, this is a stronger knowledge condition than in the previous cases. In the previous cases, there is a single newly discovered event that contains the new state, and knowledge of its probability is assumed. In the case of a new consequence, by contrast, there generally is a set of newly discovered act events that contain the new state, and their joint probability $\widehat{p}(\mathcal{A})$ cannot be deduced (in the absence of act independence or another identifying assumption) from their individual probabilities $\alpha_{i}$ (knowledge of which is assumed). The exception is the special case where

\footnotetext{
${ }^{12}$ By reverse Bayesianism, the decision maker knows $\widehat{p}(E(s))=p(s)$ and $\widehat{p}(L(s) \cup\{s\})=(1-\delta) p(s)+\widehat{p}(s)$.

${ }^{13}$ By reverse Bayesianism, the decision maker knows $\widehat{p}(C(s) \cup\{s\})=(1-\delta) p(C(s))+\widehat{p}(s)$.
} 
the new consequence is linked to a single act $f_{k}$, in which case $\widehat{p}(\mathcal{A})=\alpha_{k}$ (which is known). In this special case the value of $\widehat{p}(s)$ is determined by the roots of a quadratic equation, similar to the case of a new link.

\section{Concluding Remarks}

Reverse Bayesianism offers an elegant choice-theoretic belief revision theory that mirrors the familiar process of Bayesian updating. Reverse Bayesianism alone, however, does not fully determine the revised probability distribution over the expanded state space. The reason is that reverse Bayesianism implies restrictions on the revised probabilities of non-null states in the original state space (or, in the case of a new act, their corresponding events in the expanded state space), but not on the probabilities of new states in the expanded state space. In other words, reverse Bayesianism prescribes how probability mass shifts away from nonnull states in the original state space to the corresponding states or events in the expanded state space, but it does not prescribe how the shifted probability mass is distributed among the new states in the expanded state space.

We show that with act independence, and knowledge of the probabilities of the new act events in the expanded state space, reverse Bayesianism fully determines the revised probability distribution over the expanded state space in each case of growing awareness. While reverse Bayesianism dictates how probability mass shifts away from non-null states in the original state space, act independence dictates how the shifted probability mass is apportioned among the new states in the expanded state space. Together, reverse Bayesianism and act independence fully identify the revised probability distribution.

Without act independence, knowledge of the probabilities of the new act events in the expanded state space is no longer sufficient for reverse Bayesianism to pin down the probabilities of the new states in the expanded state space. In the case of a new act or link, we show that to pin down the probability of any new state, such knowledge must be coupled 
with knowledge of the correlation between the new act event that contains such state and the intersection of the other constituent act events for such state. In the case of a new consequence, the decision maker must know the joint probability of the newly discovered act events that contain the new state and the correlation between the intersection of such newly discovered act events and the intersection of the other constituent act events for such state.

We believe that our results may be helpful when studying economic problems where there may be unawareness. One area in which they are likely to be relevant is tort law. An injurer may cause harm to a victim because her actions have consequences of which she was previously unaware. We study this problem in Chakravarty et al. (2019). We show that under negligence when the court announces the new standard of due care this enables potential injurers to update their beliefs and learn the new probability distribution. As a result, negligence has a public good property in that it spreads awareness. Hence, in the presence of unawareness, negligence performs better than alternative rules such as strict liability. Other possible applications include regulation of technologies which damage the environment in unforeseen ways or the adoption of new technologies such as artificial intelligence, which may have unexpected outcomes, good or bad. 


\section{Appendix}

\section{Proof of Theorem 1}

(i) Take any $s \in S$. By reverse Bayesianism, we have $|S|-1$ linearly independent equations:

$$
\widehat{p}(t)=\frac{p(t)}{p(s)} \widehat{p}(s), \quad \forall t \in S, t \neq s
$$

By the definition of $\delta$ and $\sum_{t \in \widehat{S}} \widehat{p}(t)=1$, we have

$$
\sum_{t \in S} \widehat{p}(t)=1-\delta
$$

Substituting (1.1) into (1.2), we have

$$
\widehat{p}(s)+\sum_{t \in S: t \neq s} \frac{p(t)}{p(s)} \widehat{p}(s)=1-\delta,
$$

which implies

$$
\widehat{p}(s)=\frac{(1-\delta) p(s)}{\sum_{t \in S} p(t)}=(1-\delta) p(s),
$$

where the last equality follows from $\sum_{t \in S} p(t)=1$.

(ii) Take any $s \in \Delta$. By act independence,

$$
\widehat{p}(s)=\prod_{i=1}^{m} \widehat{p}\left(A_{i}\left(s^{i}\right)\right)
$$

Note that $\widehat{p}\left(A_{l}\left(s^{l}\right)\right)=\widehat{p}\left(A_{l}\left(z_{k}\right)\right)=\delta$ and $\bigcap_{i \neq l} A_{i}\left(s^{i}\right)=L(s) \cup\{s\}$. It follows that

$$
\begin{aligned}
\widehat{p}(s) & =\delta \prod_{i \neq l} \widehat{p}\left(A_{i}\left(s^{i}\right)\right)=\delta \widehat{p}\left(\bigcap_{i \neq l} A_{i}\left(s^{i}\right)\right) \\
& =\delta \widehat{p}(L(s) \cup\{s\})=\delta[\widehat{p}(L(s))+\widehat{p}(s)],
\end{aligned}
$$

which implies

$$
\widehat{p}(s)=\frac{\delta}{1-\delta} \widehat{p}(L(s))
$$

Observe that $L(s)$ is the union of all $t \in S$ such that $t^{i}=s^{i}$ for all $i \neq l$. It follows that

$$
\widehat{p}(L(s))=\sum_{t \in L(s)} \widehat{p}(t)=\sum_{t \in L(s)}(1-\delta) p(t)=(1-\delta) p(L(s)),
$$

where the second equality follows from (1.3). Substituting (1.5) back into (1.4), we have $\widehat{p}(s)=\delta p(L(s))$. 


\section{Proof of Theorem 2}

Take any $s \in S$. By reverse Bayesianism, we have $|S|-1$ linearly independent equations:

$$
p(t) \widehat{p}(E(s))=p(s) \widehat{p}(E(t)), \quad \forall t \in S, t \neq s .
$$

Summing the left- and right-hand sides, and adding $p(s) \widehat{p}(E(s))$ to each side, yields

$$
\widehat{p}(E(s)) \sum_{t \in S} p(t)=p(s) \sum_{t \in S} \widehat{p}(E(t)) .
$$

Because $\sum_{t \in S} p(t)=1$ and $\sum_{t \in S} \widehat{p}(E(t))=1$, we have

$$
\widehat{p}(E(s))=p(s)
$$

Take any $s_{j} \in E(s), j \in\{1, \ldots, n\}$. By act independence,

$$
\widehat{p}\left(s_{j}\right)=\prod_{i=1}^{m+1} \widehat{p}\left(A_{i}\left(s_{j}^{i}\right)\right)
$$

Note that $\widehat{p}\left(A_{m+1}\left(s_{j}^{m+1}\right)\right)=\widehat{p}\left(A_{m+1}\left(z_{j}\right)\right)=\delta_{j}$ and $\bigcap_{i=1}^{m} A_{i}\left(s_{j}^{i}\right)=E(s)$. It follows that

$$
\widehat{p}\left(s_{j}\right)=\delta_{j} \prod_{i=1}^{m} \widehat{p}\left(A_{i}\left(s_{j}^{i}\right)\right)=\delta_{j} \widehat{p}\left(\bigcap_{i=1}^{m} A_{i}\left(s_{j}^{i}\right)\right)=\delta_{j} \widehat{p}(E(s))
$$

Substituting (2.1) into (2.2), we have $\widehat{p}\left(s_{j}\right)=\delta_{j} p(s)$.

\section{Proof of Theorem 3}

(i) Take any $s \in S$. By reverse Bayesianism, we have $|S|-1$ linearly independent equations:

$$
p(t) \widehat{p}(s)=p(s) \widehat{p}(t), \quad \forall t \in S, t \neq s .
$$

Summing the left- and right-hand sides, and adding $p(s) \widehat{p}(s)$ to each side, yields

$$
\widehat{p}(s) \sum_{t \in S} p(t)=p(s) \sum_{t \in S} \widehat{p}(t)
$$

Observe that $\sum_{t \in S} p(t)=1$ and $\sum_{t \in S} \widehat{p}(t)=1-\delta=\prod_{i=1}^{m}\left(1-\alpha_{i}\right)$. Thus,

$$
\widehat{p}(s)=(1-\delta) p(s)=\left(\prod_{i=1}^{m}\left(1-\alpha_{i}\right)\right) p(s) .
$$


(ii) Take any $s \in \Delta$ such that $I(s)=\{k\}$ for any $k \in\{1, \ldots, m\}$. By act independence,

$$
\widehat{p}(s)=\prod_{i=1}^{m} \widehat{p}\left(A_{i}\left(s^{i}\right)\right) .
$$

Note that $\widehat{p}\left(A_{k}\left(s^{k}\right)\right)=\widehat{p}\left(A_{k}\left(z_{n+1}\right)\right)=\alpha_{k}$. Hence,

$$
\widehat{p}(s)=\alpha_{k} \prod_{i \in \bar{I}(s)} \widehat{p}\left(A_{i}\left(s^{i}\right)\right) .
$$

Observe that $I(s)=\{k\}$ implies $\bigcap_{i \in \bar{I}(s)} A_{i}\left(s^{i}\right)=C(s) \cup\{s\}$. Thus,

$$
\begin{aligned}
\widehat{p}(s) & =\alpha_{k} \prod_{i \in \bar{I}(s)} \widehat{p}\left(A_{i}\left(s^{i}\right)\right)=\alpha_{k} \widehat{p}\left(\bigcap_{i \in \bar{I}(s)} A_{i}\left(s^{i}\right)\right) \\
& =\alpha_{k} \widehat{p}(C(s) \cup\{s\})=\alpha_{k}(\widehat{p}(C(s))+\widehat{p}(s)),
\end{aligned}
$$

which implies

$$
\widehat{p}(s)=\frac{\alpha_{k}}{1-\alpha_{k}} \widehat{p}(C(s)) .
$$

Note that $C(s)$ is the union of all $t \in S$ such that $t^{i}=s^{i}$ for all $i \in \bar{I}(s)$. It follows that

$$
\widehat{p}(C(s))=\sum_{t \in C(s)} \widehat{p}(t)=\sum_{t \in C(s)}(1-\delta) p(t)=(1-\delta) p(C(s)),
$$

where the second equality follows from (3.1). Substituting (3.3) back into (3.2), we have

$$
\widehat{p}(s)=\frac{\alpha_{k}}{1-\alpha_{k}}(1-\delta) p(C(s))=\alpha_{k} \prod_{i \in \bar{I}(s)}\left(1-\alpha_{i}\right) p(C(s)),
$$

where the last equality follows from $1-\delta=\prod_{i=1}^{m}\left(1-\alpha_{i}\right)$.

Next take any $s \in \Delta$ such that $I(s)=\{k, l\}$ for any $\{k, l\} \subset\{1, \ldots, m\}$. By act independence,

$$
\widehat{p}(s)=\prod_{i=1}^{m} \widehat{p}\left(A_{i}\left(s^{i}\right)\right) .
$$

Note that $\widehat{p}\left(A_{k}\left(s^{k}\right)\right)=\widehat{p}\left(A_{k}\left(z_{n+1}\right)\right)=\alpha_{k}$. Hence,

$$
\widehat{p}(s)=\alpha_{k} \prod_{i \in\{\bar{I}(s) \cup\{l\}\}} \widehat{p}\left(A_{i}\left(s^{i}\right)\right) .
$$

Observe that $I(s)=\{k, l\}$ implies $\bigcap_{i \in\{\bar{I}(s) \cup\{l\}\}} A_{i}\left(s^{i}\right)=D(s) \cup\{s\}$, where $D(s) \equiv\{r \in \Delta$ : $r^{i}=s^{i}, \forall i \in\{\bar{I}(s) \cup\{l\}\}$. Thus,

$$
\begin{aligned}
\widehat{p}(s) & =\alpha_{k} \prod_{i \in\{\bar{I}(s) \cup\{l\}\}} \widehat{p}\left(A_{i}\left(s^{i}\right)\right)=\alpha_{k} \widehat{p}\left(\bigcap_{i \in\{\bar{I}(s) \cup\{l\}} A_{i}\left(s^{i}\right)\right) \\
& =\alpha_{k} \widehat{p}(D(s) \cup\{s\})=\alpha_{k}(\widehat{p}(D(s))+\widehat{p}(s)),
\end{aligned}
$$


which implies

$$
\widehat{p}(s)=\frac{\alpha_{k}}{1-\alpha_{k}} \widehat{p}(D(s)) .
$$

Observe further that $I(r)=\{l\}$ for all $r \in D(s)$. It follows that

$$
\begin{aligned}
\widehat{p}(D(s)) & =\sum_{t \in D(s)} \widehat{p}(t) \\
& =\sum_{t \in D(s)} \frac{\alpha_{l}}{1-\alpha_{l}}(1-\delta) p(C(t)) \\
& =\frac{\alpha_{l}}{1-\alpha_{l}}(1-\delta) p(C(s)) .
\end{aligned}
$$

Substituting (3.5) back into (3.4), we have

$$
\begin{aligned}
\widehat{p}(s) & =\frac{\alpha_{k}}{1-\alpha_{k}} \frac{\alpha_{l}}{1-\alpha_{l}}(1-\delta) p(C(s)) . \\
& =\alpha_{k} \alpha_{l} \prod_{i \in \bar{I}(s)}\left(1-\alpha_{i}\right) p(C(s))
\end{aligned}
$$

Proceeding in this fashion to consider $s \in \Delta$ such that $I(s)$ is an $\iota$-element subset of $\{1, \ldots, m\}$ for all $\iota=3, \ldots, m-1$, we establish that

$$
\widehat{p}(s)=\left(\prod_{i \in I(s)} \alpha_{i}\right)\left(\prod_{i \in \bar{I}(s)}\left(1-\alpha_{i}\right)\right) p(C(s))
$$

for all $s \in \Delta$ such that $I(s) \subset\{1, \ldots, m\}$.

(iii) Take the $s \in \Delta$ such that $I(s)=\{1, \ldots, m\}$. By act independence, $\widehat{p}(s)=$ $\prod_{i=1}^{m} \widehat{p}\left(A_{i}\left(s^{i}\right)\right)$. Observe that $\widehat{p}\left(A_{i}\left(s^{i}\right)\right)=\widehat{p}\left(A_{i}\left(z_{n+1}\right)\right)=\alpha_{i}$ for all $i \in I(s)$. Because $I(s)=\{1, \ldots, m\}$, we have $\widehat{p}(s)=\prod_{i=1}^{m} \alpha_{i}$.

\section{Proof of Theorem 4}

Take any $s \in S$ and corresponding $E(s) \subset \widehat{S}$. For any $s_{j} \in E(s), \widehat{p}\left(s_{j}\right)=\widehat{p}\left(\bigcap_{i=1}^{m+1} A_{i}\left(s^{i}\right)\right)$. By definition, $\Delta_{j}=A_{m+1}\left(z_{j}\right)$ and $E(s)=\bigcap_{i=1}^{m} A_{i}\left(s_{j}^{i}\right)$. Let $\rho\left(\Delta_{j}, E(s)\right)$ denote the correlation between $\Delta_{j}$ and $E(s)$. It follows that

$$
\begin{aligned}
\widehat{p}\left(s_{j}\right) & =\widehat{p}\left(\bigcap_{i=1}^{m+1} A_{i}\left(s^{i}\right)\right)=\widehat{p}\left(\Delta_{j} \cap E(s)\right) \\
& =\widehat{p}\left(\Delta_{j}\right) \widehat{p}(E(s))+\rho\left(\Delta_{j}, E(s)\right) \sqrt{\widehat{p}\left(\Delta_{j}\right)\left(1-\widehat{p}\left(\Delta_{j}\right)\right) \widehat{p}(E(s))(1-\widehat{p}(E(s)))}
\end{aligned}
$$

where the last line follows from the fact that, for two events $A$ and $B$,

$$
\rho(A, B)=\frac{P(A \cap B)-P(A) P(B)}{\sqrt{P(A)(1-P(A))} \sqrt{P(B)(1-P(B))}} .
$$


By definition, $\delta_{j}=\widehat{p}\left(\Delta_{j}\right)$. By reverse Bayesianism, $\widehat{p}(E(s))=p(s)$ (see Theorem 2). Hence,

$$
\widehat{p}\left(s_{j}\right)=\delta_{j} p(s)+\rho\left(\Delta_{j}, E(s)\right) \sqrt{\delta_{j}\left(1-\delta_{j}\right) p(s)(1-p(s))} .
$$

\section{Proof of Theorem 5}

Take any $s \in \Delta$. Recall that $\widehat{p}(s)=\widehat{p}\left(\bigcap_{i=1}^{m} A_{i}\left(s^{i}\right)\right)$. By definition, $\Delta=A_{l}\left(z_{k}\right)$ and $L(s) \cup\{s\}=\bigcap_{i \neq l} A_{i}\left(s^{i}\right)$. Let $\rho=\rho(\Delta, L(s) \cup\{s\})$ denote the correlation between $\Delta$ and $L(s) \cup\{s\}$. It follows that

$$
\begin{aligned}
\widehat{p}(s) & =\widehat{p}\left(\bigcap_{i=1}^{m} A_{i}\left(s^{i}\right)\right)=\widehat{p}(\Delta \cap(L(s) \cup\{s\})) \\
& =\widehat{p}(\Delta) \widehat{p}(L(s) \cup\{s\})+\rho \sqrt{\widehat{p}(\Delta)(1-\widehat{p}(\Delta)) \widehat{p}(L(s) \cup\{s\})(1-\widehat{p}(L(s) \cup\{s\}))} \\
& =\widehat{p}(\Delta)[\widehat{p}(L(s))+\widehat{p}(s)]+\rho \sqrt{\widehat{p}(\Delta)(1-\widehat{p}(\Delta))[\widehat{p}(L(s))+\widehat{p}(s)](1-[\widehat{p}(L(s))+\widehat{p}(s)])},
\end{aligned}
$$

where the second line follows from (4.1) and the last line follows because $L(s) \cap\{s\}=\emptyset$. By definition, $\delta=\widehat{p}(\Delta)$. By reverse Bayesianism, $\widehat{p}(L(s))=(1-\delta) p(L(s))$ (see Theorem 1). Thus,

$$
\begin{aligned}
\widehat{p}(s)=\delta[(1-\delta) p(L(s))+\widehat{p}(s)] & \\
& +\rho \sqrt{\delta(1-\delta)[(1-\delta) p(L(s))+\widehat{p}(s)](1-[(1-\delta) p(L(s))+\widehat{p}(s)])} .
\end{aligned}
$$

Equation (5.1) implies that $\widehat{p}(s)$ is determined by the roots of a quadratic equation. The following lemmas, which complete the proof, (i) derive the quadratic equation, (ii) show that its roots are real and unequal, (iii) show that both roots are less than one; (iv) show that the larger root is positive and when the smaller root is positive, (v) derive the roots, and (vi) show that the larger root applies when $\rho>0$ and the smaller root applies when $\rho<0$.

Lemma 1. $\widehat{p}(s)$ is a root of the quadratic equation $a x^{2}+b x+c=0$, where $x=\widehat{p}(s)$, $y=p(L(s))$,

$$
\begin{aligned}
a & =1-\delta+\delta \rho^{2}>0, \\
b & =-\left[2 y \delta(1-\delta)\left(1-\rho^{2}\right)+\delta \rho^{2}\right]<0, \text { and } \\
c & =y \delta(1-\delta)\left[y \delta\left(1-\rho^{2}\right)-\rho^{2}(1-y)\right] .
\end{aligned}
$$

Proof. Substituting $x=\widehat{p}(s)$ and $y=p(L(s))$ into (5.1), we have

$$
x=\delta[(1-\delta) y+x]+\rho \sqrt{\delta(1-\delta)[(1-\delta) y+x](1-[(1-\delta) y+x])} .
$$


It follows that:

$$
\begin{aligned}
& {[(1-\delta) x-\delta(1-\delta) y]^{2}=\rho^{2} \delta(1-\delta)\left[(1-\delta) y+x-((1-\delta) y+x)^{2}\right] } \\
\Rightarrow \quad & (1-\delta)^{2}(x-\delta y)^{2}=\rho^{2} \delta(1-\delta)^{2} y+\rho^{2} \delta(1-\delta) x-\rho^{2} \delta(1-\delta)((1-\delta) y+x)^{2} \\
\Rightarrow \quad & (1-\delta)\left(x^{2}-2 \delta y x+\delta^{2} y^{2}\right)-\rho^{2} \delta(1-\delta) y-\rho^{2} \delta x+\rho^{2} \delta\left((1-\delta)^{2} y^{2}+2(1-\delta) y x+x^{2}\right)=0 \\
\Rightarrow \quad & {\left[1-\delta+\rho^{2} \delta\right] x^{2}+\left[2 \rho^{2} \delta(1-\delta) y-2 \delta(1-\delta) y-\rho^{2} \delta\right] x+(1-\delta) \delta^{2} y^{2}-\rho^{2} \delta(1-\delta) y+\rho^{2} \delta(1-\delta)^{2} y^{2}=0 } \\
\Rightarrow \quad & {\left[1-\delta+\delta \rho^{2}\right] x^{2}-\left[2 y \delta(1-\delta)\left(1-\rho^{2}\right)+\delta \rho^{2}\right] x+y \delta(1-\delta)\left[y \delta\left(1-\rho^{2}\right)-\rho^{2}(1-y)\right]=0 . }
\end{aligned}
$$

Lemma 2. The roots of the quadratic equation in Lemma 1 are real and unequal.

Proof. Let $a x^{2}+b x+c=0$ be the quadratic equation in in Lemma 1. The roots are real and unequal if $b^{2}-4 a c>0$. Substiuting the expressions for $a, b$, and $c$, we have

$$
\begin{aligned}
b^{2}-4 a c & =\left[-2 \delta(1-\delta) y\left(1-\rho^{2}\right)-\delta \rho^{2}\right]^{2}-4\left(1-\delta+\delta \rho^{2}\right)\left(\delta(1-\delta) y\left[y \delta\left(1-\rho^{2}\right)-\rho^{2}(1-y)\right]\right) \\
& =\delta^{2}\left[2 y-2 y \delta+\rho^{2}-2 y \rho^{2}+2 y \delta \rho^{2}\right]^{2}-4\left(1-\delta+\delta \rho^{2}\right)\left(\delta(1-\delta) y\left(y \delta-\rho^{2}+y \rho^{2}-y \delta \rho^{2}\right)\right) \\
& =\delta \rho^{2}\left(4 y-4 y \delta+4 y^{2} \delta+\delta \rho^{2}-4 y^{2}\right) \\
& =\delta \rho^{2}\left(4 y(1-\delta)(1-y)+\delta \rho^{2}\right)>0
\end{aligned}
$$

where the inequality follows because $\delta, y \in(0,1)$.

Lemma 3. Both roots of the quadratic equation in Lemma 1 are less than one.

Proof. Let $a x^{2}+b x+c=0$ be the quadratic equation in Lemma 1. Both roots are less than one if the larger root is less than one, i.e., if $-b+\sqrt{b^{2}-4 a c}<2 a$. (Recall that $a>0$.) Observe that

$$
\begin{aligned}
-b+\sqrt{b^{2}-4 a c}<2 a & \Leftrightarrow 2 a+b>\sqrt{b^{2}-4 a c} \\
& \Leftrightarrow 4 a^{2}+4 a b+4 a c>0 \\
& \Leftrightarrow a+b+c>0 .
\end{aligned}
$$

Substiuting the expressions for $a, b$, and $c$, we have

$$
\begin{aligned}
a+b+c & =1-\delta+\delta \rho^{2}-2 y \delta(1-\delta)\left(1-\rho^{2}\right)-\delta \rho^{2}+y \delta(1-\delta)\left[y \delta\left(1-\rho^{2}\right)-\rho^{2}(1-y)\right] \\
& =1-\delta-2 y \delta(1-\delta)\left(1-\rho^{2}\right)+y^{2} \delta^{2}(1-\delta)\left(1-\rho^{2}\right)-y \delta \rho^{2}(1-\delta)(1-y) \\
& =(1-\delta)\left[1-2 y \delta\left(1-\rho^{2}\right)+y^{2} \delta^{2}\left(1-\rho^{2}\right)-y \delta \rho^{2}(1-y)\right] \\
& =(1-\delta)\left[1-2 y \delta+y \delta \rho^{2}+y^{2} \delta^{2}-y^{2} \delta^{2} \rho^{2}+y^{2} \delta \rho^{2}\right] \\
& =(1-\delta)\left[\left(1-2 y \delta+y^{2} \delta^{2}\right)+y^{2} \delta \rho^{2}+\left(y \delta \rho^{2}-y^{2} \delta^{2} \rho^{2}\right)\right] \\
& =(1-\delta)\left[(1-y \delta)^{2}+y^{2} \delta \rho^{2}+y \delta \rho^{2}(1-y \delta)\right]>0,
\end{aligned}
$$

where the inequality follows because $\delta, y \in(0,1)$. 
Lemma 4. Let $a x^{2}+b x+c=0$ be the quadratic equation in Lemma 1. Let the roots be $r_{1}>r_{2}$. Then $r_{1}>0, r_{2}>0$ if $c>0$, and $r_{2}<0$ if $c<0$.

Proof. If the roots are $r_{1}>r_{2}$, then $\left(x-r_{1}\right)\left(x-r_{2}\right)=x^{2}-\left(r_{1}+r_{2}\right) x+r_{1} r_{2}=0$. Note $a x^{2}+b x+c=0$ implies $x^{2}+\frac{b}{a} x+\frac{c}{a}=0$. Hence $\frac{b}{a}=-\left(r_{1}+r_{2}\right)$ and $\frac{c}{a}=r_{1} r_{2}$. Recall $a>0$ and $b<0$. Thus $\frac{b}{a}=-\left(r_{1}+r_{2}\right)$ implies $r_{1}+r_{2}>0$. This in turn implies $r_{1}>0$ (because $r_{1}>r_{2}$ ). Because $\frac{c}{a}=r_{1} r_{2}$ and $a>0, r_{1}>0$ implies $r_{2}>0$ if $c>0$ and $r_{2}<0$ if $c<0$.

Remark. Given the expression for $c$, Lemma 4 establishes a necessary and sufficient condition for $r_{2} \geq 0:-\frac{\delta y}{1-y+\delta y} \leq \rho \leq+\frac{\delta y}{1-y+\delta y}$. Observe that the bounds go to zero as $\delta$ or $y$ goes to zero, go to $-y$ and $+y$ as $\delta$ goes to one, and go to -1 and +1 as $y$ goes to one. This is what we would expect, and we conjecture that the condition is satisfied for admissible combinations of $\delta, y$, and $\rho$.

Lemma 5. Let $a x^{2}+b x+c=0$ be the quadratic equation in Lemma 1. Then the roots are

$$
r_{1}=\delta y+\frac{\delta \rho^{2}}{2\left(1-\delta+\delta \rho^{2}\right)}\left[1-2 y+\sqrt{1+\left(4 y(1-\delta)(1-y) / \delta \rho^{2}\right)}\right]
$$

and

$$
r_{2}=\delta y+\frac{\delta \rho^{2}}{2\left(1-\delta+\delta \rho^{2}\right)}\left[1-2 y-\sqrt{1+\left(4 y(1-\delta)(1-y) / \delta \rho^{2}\right)}\right] .
$$

Moreover, $r_{1}>\delta y>r_{2}$.

Proof. Using the expressions for $a$ and $b$ from Lemma 1 and the expression for $b^{2}-4 a c$ from Lemma 2, the larger root is given by

$$
\begin{aligned}
r_{1}=\frac{-b+\sqrt{b^{2}-4 a c}}{2 a} & =\frac{2 y \delta(1-\delta)\left(1-\rho^{2}\right)+\delta \rho^{2}+\sqrt{\delta \rho^{2}\left(4 y(1-\delta)(1-y)+\delta \rho^{2}\right)}}{2\left(1-\delta+\delta \rho^{2}\right)}, \\
& =\frac{2 y \delta(1-\delta)+2 y \delta^{2} \rho^{2}}{2\left(1-\delta+\delta \rho^{2}\right)}+\frac{\delta \rho^{2}-2 y \delta \rho^{2}+\delta \rho^{2} \sqrt{1+\left(4 y(1-\delta)(1-y) / \delta \rho^{2}\right)}}{2\left(1-\delta+\delta \rho^{2}\right)} \\
& =\delta y+\frac{\delta \rho^{2}}{2\left(1-\delta+\delta \rho^{2}\right)}\left[1-2 y+\sqrt{1+\left(4 y(1-\delta)(1-y) / \delta \rho^{2}\right)}\right] .
\end{aligned}
$$

Similarly, the smaller root is given by

$$
\begin{aligned}
r_{2}=\frac{-b-\sqrt{b^{2}-4 a c}}{2 a} & =\frac{2 y \delta(1-\delta)\left(1-\rho^{2}\right)+\delta \rho^{2}-\sqrt{\delta \rho^{2}\left(4 y(1-\delta)(1-y)+\delta \rho^{2}\right)}}{2\left(1-\delta+\delta \rho^{2}\right)}, \\
& =\frac{2 y \delta(1-\delta)+2 y \delta^{2} \rho^{2}}{2\left(1-\delta+\delta \rho^{2}\right)}+\frac{\delta \rho^{2}-2 y \delta \rho^{2}-\delta \rho^{2} \sqrt{1+\left(4 y(1-\delta)(1-y) / \delta \rho^{2}\right)}}{2\left(1-\delta+\delta \rho^{2}\right)} \\
& =\delta y+\frac{\delta \rho^{2}}{2\left(1-\delta+\delta \rho^{2}\right)}\left[1-2 y-\sqrt{1+\left(4 y(1-\delta)(1-y) / \delta \rho^{2}\right)}\right] .
\end{aligned}
$$


Note that $r_{1}>\delta y \Leftrightarrow 1-2 y+\sqrt{\left(4 y(1-\delta)(1-y) / \delta \rho^{2}+1\right)}>0$, which is true:

$$
\begin{aligned}
& 1-2 y+\sqrt{\left(4 y(1-\delta)(1-y) / \delta \rho^{2}+1\right)}>0 \\
\Leftrightarrow & 4 y(1-\delta)(1-y) / \delta \rho^{2}>(2 y-1)^{2}-1 \\
\Leftrightarrow & 4 y(1-\delta)(1-y) / \delta \rho^{2}>4 y(y-1),
\end{aligned}
$$

which is true because $\delta, y \in(0,1)$. A similar argument establishes that $r_{2}<\delta y$.

Lemma 6. Let $a x^{2}+b x+c=0$ be the quadratic equation in Lemma 1. Let the roots be $r_{1}>r_{2}$. Then $x=r_{1}$ if $\rho>0$ and $x=r_{2}$ if $\rho<0$.

Proof. By (5.2),

$$
\rho=\frac{x-\delta[(1-\delta) y+x]}{\sqrt{\delta(1-\delta)[(1-\delta) y+x](1-[(1-\delta) y+x])}} .
$$

Assume $\rho>0$. This implies $x>\delta[(1-\delta) y+x]$, which in turn implies $x>\delta y$. By Lemma $5, r_{1}>\delta y>r_{2}$. Hence, $x=r_{1}$ if $\rho>0$. A similar argument establishes that $x=r_{2}$ if $\rho<0$. 


\section{References}

Becker, C. K., Melkonyan, T., Proto, E., Sofianos, A., and Trautmann, S. T. "Reverse Bayesianism: Revising Beliefs in Light of Unforeseen Events." CESifo Working Paper No. 8662, 2021.

Chakravarty, S., Kelsey, D., and Teitelbaum, J. C. "Tort Liability and Unwareness." SSRN Working Paper No. 3179753, 2019.

Chambers, C. P. and Hayashi, T. "Reverse Bayesianism: A Comment." American Economic Journal: Microeconomics, Vol. 10 (2018), pp. 315-324.

Collee, J. G. and Bradley, R. "BSE: A Decade On-Part I." The Lancet, Vol. 349 (1997), pp. 636-641.

Dekel, E., Lipman, B. L., and Rustichini, A. "Recent Developments in Modeling Unforeseen Contingencies." European Economic Review, Vol. 42 (1998a), pp. 523-542.

Dekel, E., Lipman, B. L., and Rustichini, A. "Standard State-Space Models Preclude Unawareness." Econometrica, Vol. 66 (1998b), pp. 159-173.

Dominiak, A. and Tserenjigmid, G. "Belief Consistency and Invariant Risk Preferences." Journal of Mathematical Economics, Vol. 79 (2018), pp. 157-162.

Dominiak, A. and Tserenjigmid, G. "Ambiguity under Growing Unawareness." Journal of Economic Theory, 2021, in press.

Fagin, R. and Halpern, J. Y. "Belief, Awareness, and Limited Reasoning." Artificial Intelligence, Vol. 34 (1988), pp. 39-76.

Gold, R. The Boom: How Fracking Ignited the American Energy Revolution and Changed the World. New York: Simon \& Schuster, 2014.

Grant, S., Meneghel, I., and Tourky, R. "Learning under Unawareness." Working Paper, Research School of Economics, Australian National University, 2019.

Halpern, J. Y. "Alternative Semantics for Unawareness." Games and Economic Behavior, Vol. 37 (2001), pp. 321-339.

Halpern, J. Y. and Rêgo, L. C. "Interactive Unawareness Revisited." Games and Economic Behavior, Vol. 62 (2008), pp. 232-262.

Heifetz, A., Meier, M., and Schipper, B. C. "Interactive Unawareness." Games and Economic Behavior, Vol. 130 (2006), pp. 78-94. 
Karni, E., Valenzuela-Stookey, Q., and Vierø, M.-L. "Reverse Bayesianism: A Generalization." B.E. Journal of Theoretical Economics, Vol. 21 (2021), pp. 557-569.

Karni, E. and Vierø, M.-L. "Reverse Bayesianism: A Choice-Based Theory of Growing Awareness." American Economic Review, Vol. 103 (2013), pp. 2790-2810.

Karni, E. and Vierø, M.-L. "Probabilistic Sophistication and Reverse Bayesianism." Journal of Risk and Uncertainty, Vol. 50 (2015), pp. 189-208.

Karni, E. and Vierø, M.-L. "Awareness of Unawareness: A Theory of Decision Making in the Face of Ignorance." Journal of Economic Theory, Vol. 168 (2017), pp. 301-328.

Lindsley, C. W. "Chronic Traumatic Encephalopathy (CTE): A Brief Historical Overview and Recent Focus on NFL Players." ACS Chemical Neuroscience, Vol. 8 (2017), pp. 16291631 .

Modica, S. and Rustichini, A. "Awareness and Partitional Information Structures." Theory and Decision, Vol. 27 (1994), pp. 265-298.

Modica, S. and Rustichini, A. "Unawareness and Partitional Information Structures." Games and Economic Behavior, Vol. 37 (1999), pp. 107-124.

National Academies of Sciences, Engineering, and Medicine. "Veterans and Agent Orange: Update 11." Washington, DC: National Academic Press, 2018.

Savage, L. J. The Foundations of Statistics. New York: Wiley, 1954.

Schipper, B. C. "Preference-Based Unawareness." Mathematical Social Sciences, Vol. 70 (2014a), pp. 1-9.

Schipper, B. C. "Unawareness - A Gentle Introduction to Both the Literature and the Special Issue." Mathematical Social Sciences, Vol. 70 (2014b), pp. 34-41.

Schipper, B. C. "Awareness." In H. van Ditmarsh, J. Y. Halpern, W. van der Hock, and B. Kooi, eds., Handbook of Epistemic Logic. London: College Publications, 2015.

Sklar, A. "Random Variables, Joint Distribution Functions, and Copulas." Kybernetika, Vol. 9 (1973), pp. 449-460.

U.S. Centers for Disease Control and Prevention. "Thirty Years of HIV - 1981-2011." Morbidity and Mortality Weekly Report, Vol. 60, No. 21 (2011), p. 689.

Zheng, J. "SARS-CoV-2: An Emerging Coronavirus that Causes a Global Threat." International Journal of Biological Sciences, Vol. 16 (2020), pp. 1678-1685. 